\title{
ICONICITY IN SIGN LANGUAGE
}

\author{
KAREN EMMOREY ${ }^{* 1}$ \\ *Corresponding Author: kemmorey@mail.sdsu.edu \\ ${ }^{1}$ San Diego State University, California, USA
}

Iconicity may have played a role in language evolution under the assumption that the earliest vocal or manual communications involved motivated forms. The study of sign languages may provide some insight into this issue because the visual-manual modality allows for iconic expression of a wide range of conceptual elements, e.g., object and human actions, movements, locations, and shapes. Currently, however, we know very little about how visual-manual iconicity is perceived by signers vs. non-signers or whether iconic signs are processed differently in the brain. My colleagues and I have been exploring the nature of the distribution of iconic forms in the American Sign Language (ASL) lexicon, how the perception of iconicity is impacted by linguistic knowledge, and how the perception of iconicity changes when the sign meaning is given versus when it must be guessed. We have also been investigating whether there are general principles of alignment between visual-manual articulations and conceptual elements (e.g., handshapes map to objects). Finally, we have been using Event-Related Potentials (ERPs) to investigate the possible role of iconicity in modulating the temporal neural dynamics of single sign processing. Thus far, the ERP results indicate that iconic signs do not have a distinct neural signature, but frequency and concreteness effects are parallel for word and sign recognition. Overall, this work indicates a) an important distinction between iconicity and transparency, b) linguistic knowledge reduces and changes sensitivity to iconicity, and c) there appears to be no distinct neural response to iconic signs. 